\title{
Spontaneous biloma: a case report
}

\author{
V. Della Valle $\cdot$ E. Eshja $\cdot$ E. M. Bassi
}

Received: 29 August 2013/ Accepted: 30 October 2013/Published online: 12 November 2013

(C) Società Italiana di Ultrasonologia in Medicina e Biologia (SIUMB) 2013

\begin{abstract}
A biloma is an encapsulated collection of bile located in the abdomen. It occurs spontaneously or secondary to traumatic or iatrogenic injury to the biliary system. The patient's medical history, symptoms and diagnostic imaging findings suggest the diagnosis, but a definitive diagnosis is provided by drainage and biochemical analysis of the fluid. We report a case of a patient admitted with acute abdominal pain in the right hypochondrium caused by a spontaneous biloma. This is a rare condition, and the reason for the onset was not identified. We discuss the role of the various diagnostic imaging techniques, particularly that of ultrasound.
\end{abstract}

Keywords Spontaneous biloma · Imaging ·

Ultrasound $\cdot$ Contrast-enhanced CT

Riassunto Il biloma è una raccolta circoscritta di bile in addome, spontanea o secondaria ad una lesione traumatica o iatrogena del sistema biliare. L'anamnesi, i sintomi ed i reperti radiologici suggeriscono la diagnosi, anche se spesso la diagnosi finale è legata al drenaggio del liquido. Riportiamo il caso di un paziente ricoverato per dolore addominale acuto in ipocondrio destro determinato da un biloma spontaneo, condizione rara in cui non si identifica la causa del danno al sistema biliare e discutiamo il ruolo delle varie metodiche di diagnostica per immagine, in particolare dell'ecografia.

V. Della Valle $(\varangle) \cdot$ E. Eshja · E. M. Bassi

Department of Radiology, IRCCS Policlinico San Matteo Pavia, University of Pavia, Piazzale Golgi 1, 27100 Pavia, Italy

e-mail:vdv.valeriadellavalle@gmail.com

\section{Introduction}

A biloma is an encapsulated collection of bile located in the abdomen; it can be either intra- or extrahepatic and occurs spontaneously or secondary to a traumatic or iatrogenic lesion to the biliary system. The first cases described in the literature by Whipple [1] in 1898 and by Gould [2] in 1979 occurred secondary to blunt trauma. However, biloma more often occurs due to iatrogenic damage to the biliary system in connection with laparoscopic cholecystectomy [3]. When a specific cause is not identified, the lesion is referred to as a spontaneous biloma.

Ultrasound (US) imaging is often the first imaging modality employed in the work up of patients with abdominal pain. US imaging permits identification of free fluid in the abdominal cavity or fluid-filled sacs of a volume of $40 \mathrm{cc}$ or greater including bile collections thereby suggesting the diagnosis of biloma. However, to increase diagnostic certainty it is necessary to employ second-level methods such as contrast-enhanced computed tomography (CT) or magnetic resonance (MR) cholangiography.

We present a case of spontaneous biloma in a patient who underwent abdominal $\mathrm{X}$-ray, US and contrastenhanced CT.

\section{Description of the case}

A 79-year-old female patient was admitted with acute abdominal pain located in the right hypochondrium, associated with nausea. On physical examination, Blumberg's sign and Giordano's maneuver were negative, there was no fever or jaundice, and outcome of laboratory tests was not significant. The patient's medical history was negative for trauma and abdominal surgery. 


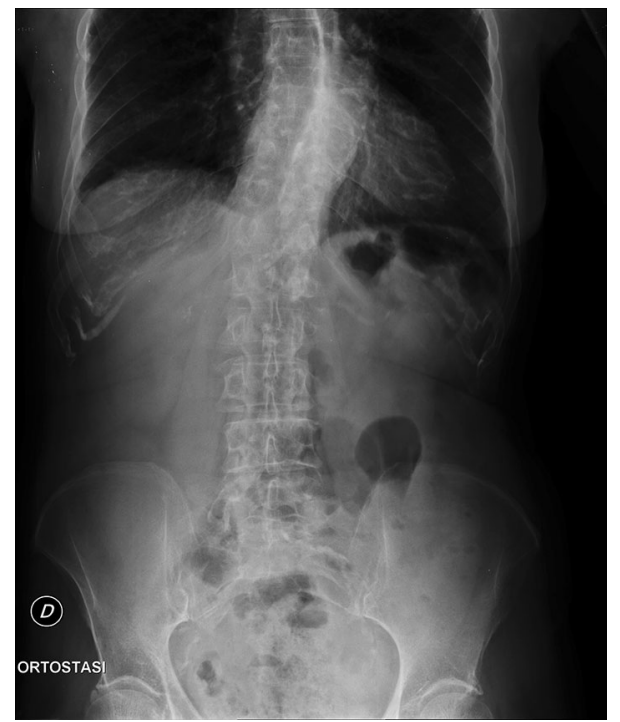

Fig. 1 X-ray of the abdomen revealed moderate elevation of the right hemidiaphragm
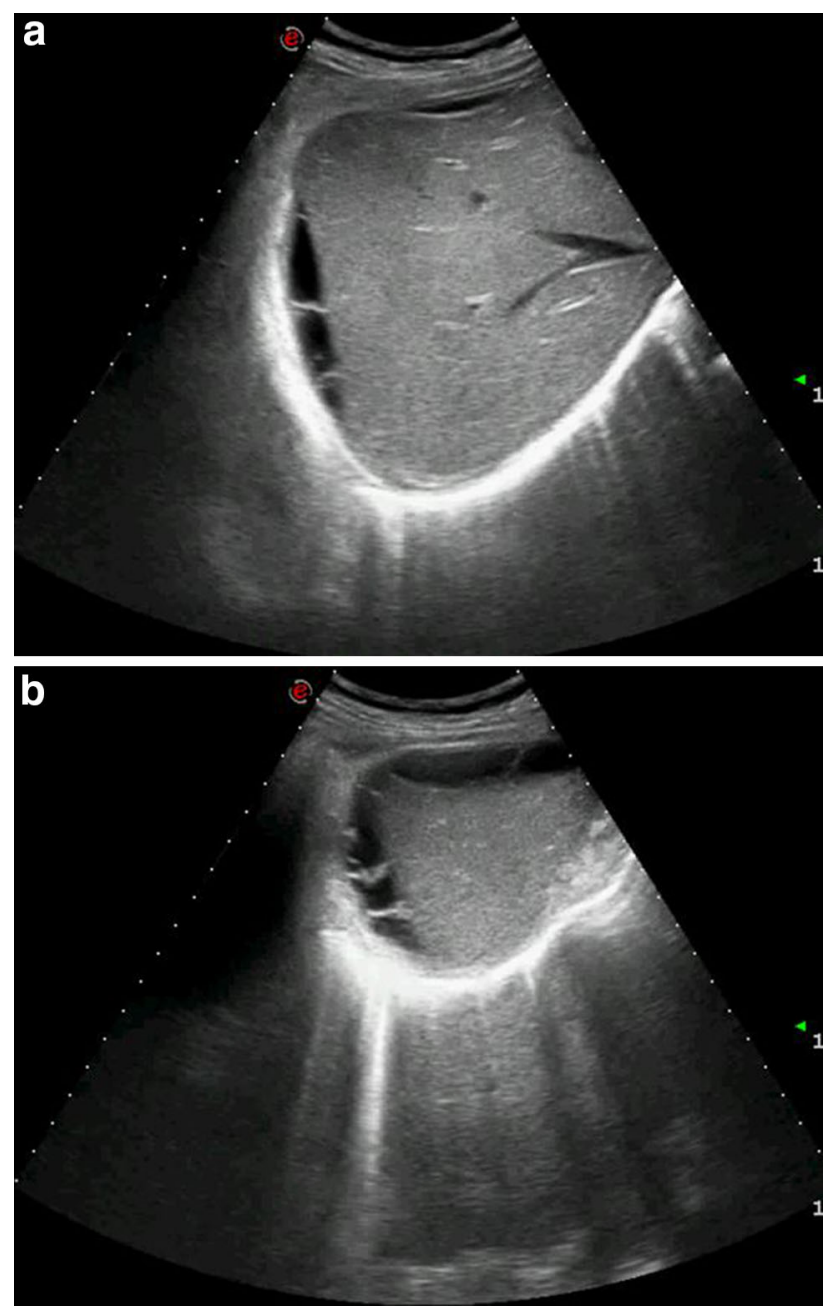

Fig. 2 a, b Gray-scale US shows a perihepatic, pluriconcamerate fluid collection
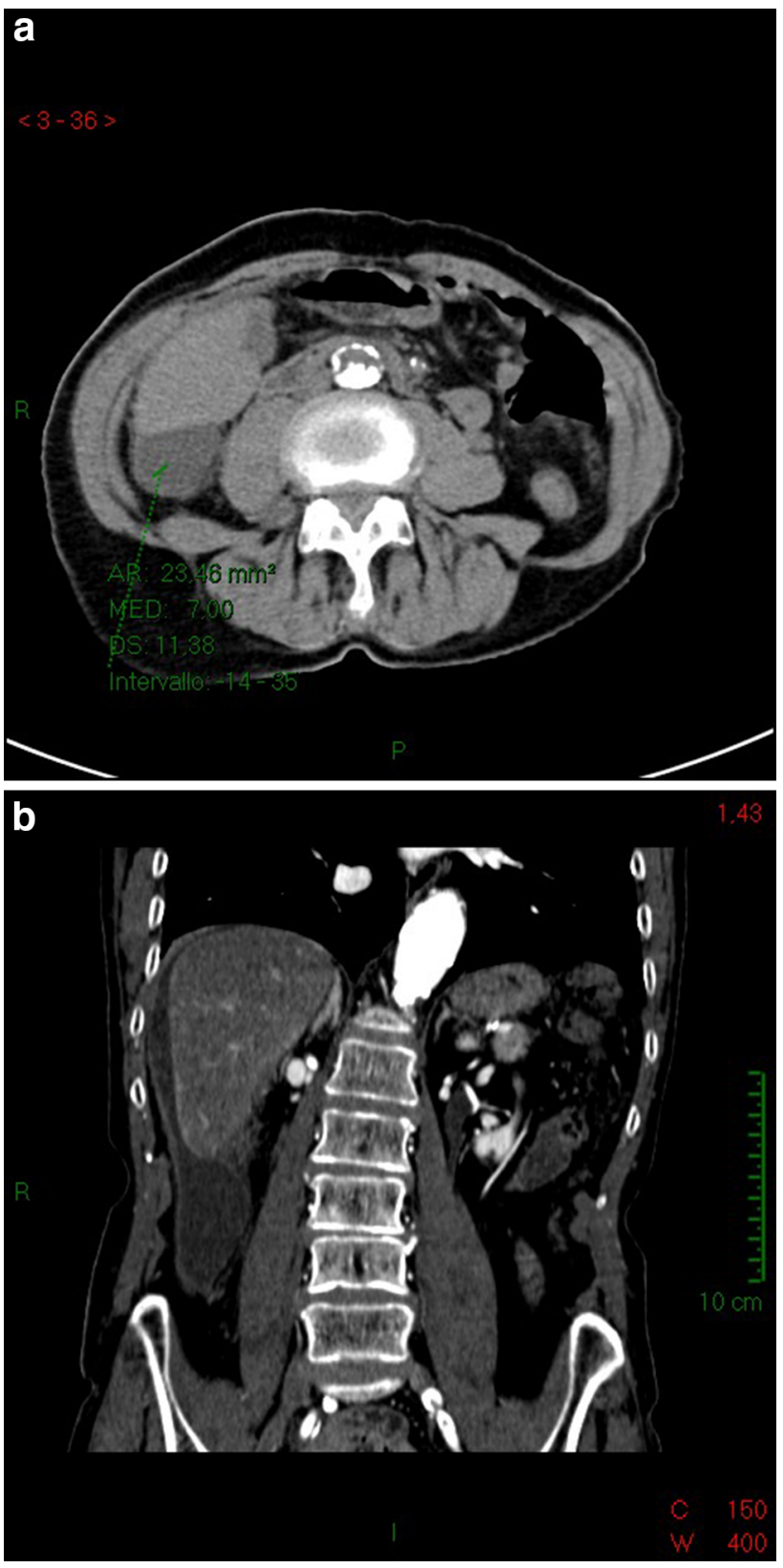

Fig. 3 a, b Abdominal CT before and after intravenous administration of contrast agent. In the axial plane CT evidences a wellcircumscribed fluid collection with well-defined margins located in the peri- and subhepatic area, with pluriconcamerate appearance. Baseline density is about $7 \mathrm{HU}$ (a) and in the coronal plane the lesion does not present significant contrast enhancement in the portal scan acquired $90 \mathrm{~s}$ after intravenous administration of contrast agent (b); there are no solid components

The patient underwent abdominal X-ray examination which showed only a slight elevation of the right hemidiaphragm (Fig. 1).

As cholelithiasis or acute cholecystitis was suspected, abdominal US imaging was carried out revealing a pluriconcamerate fluid collection with septa about 2-mm thick (Fig. 2a, b) located in the perihepatic area. Color Doppler US 
showed no vascular involvement. Due to these findings, the differential diagnosis included several diseases: biloma, lymphocele, abscess, pseudo cyst, hematoma and cystic peritoneal metastasis (e.g., originating from ovarian cancer).

Given the non-specific US findings, contrast-enhanced CT was performed confirming the presence of an encapsulated fluid collection, well defined and with pluriconcamerate appearance, located in the peri- and subhepatic area. Max axial diameter in the subhepatic area $4 \times 6 \mathrm{~cm}$, baseline density about 7 Hounsfield (HU) and no significant contrast enhancement in the various phases acquired after intravenous administration of contrast medium (8 HU in the arterial phase and $12 \mathrm{HU}$ in the portal phase $90 \mathrm{~s}$ after administration of contrast material); there were no solid components (Fig. 3).

These characteristic imaging findings and the site of the lesion supported a tentative diagnosis of biloma. Diagnosis was confirmed by analysis of the aspirated fluid. After percutaneous drainage and antibiotic therapy, the lesion disappeared and the patient obtained complete resolution of symptoms.

\section{Discussion}

The first case of biloma was described by Whipple in 1898 , but the term "biloma" was coined by Gould et al. in 1979 to describe an extrahepatic post-traumatic bile collection. Today a biloma is defined as an encapsulated collection of bile located in the abdomen; it can be either intra- or extrahepatic and it may occur spontaneously or secondary to a traumatic or iatrogenic lesion to the biliary system.

Iatrogenic injury to the biliary system is more frequent in connection with laparoscopic cholecystectomy than laparotomic cholecystectomy. Mean time for developing postcholecystectomy biloma is about 2 weeks from the surgery, but in the literature up to 5 years from the surgery has been reported [4]. Other iatrogenic causes of biloma include percutaneous transhepatic cholangiography, biopsy of the liver, liver resection, endoscopic retrograde cholangiopancreatography (ERCP) and liver drainage procedures. In rare cases when the cause is not identified, the lesion is referred to as a spontaneous biloma. In some cases, biloma has been reported in connection with biliary obstruction by gallstones, cholangiocarcinoma, acute cholecystitis, hepatic infarction, hepatic abscess, tuberculosis and sickle cell disease [5].

Regardless of etiology, clinical presentation is similar with non-specific symptoms, such as a feeling of abdominal heaviness, mild to moderate abdominal pain localized in the right hypochondrium and gradual onset of malaise and nausea. Depending on the associated pathology, other symptoms may occur, such as fever (if cholecystitis) or jaundice (if cholelithiasis). Laboratory tests are entirely non-specific and may be linked to the associated pathology (neutrophilic leukocytosis, increased indices suggesting cholestasis) [3].

Ultrasound is often employed as a first-level imaging method, because it is non-invasive, fast and easy to perform. US findings suggesting biloma are a hypo-anechoic fluid collection, well-defined margins, sometimes encapsulated, mono- or pluriconcamerate appearance, in a typical location (right upper abdominal quadrant: sub- or intrahepatic, below the diaphragm) and no vascularity on color Doppler US. The size of a biloma may vary from a few centimeters up to $40 \mathrm{~cm}$ in diameter [6].

However, these US findings are non-specific, as they occur also in other diseases which must, therefore, be included in the differential diagnosis: lymphocele, abscess, hematoma, pseudocyst, hepatic cyst, cystic seroma and cystic peritoneal metastasis (e.g., originating from ovarian cancer).

It is, therefore, necessary to employ a second-level imaging method, such as contrast-enhanced CT which permits detection of smaller bilomas characterized by welldefined margins, mono- or pluriconcamerate appearance, hypodense with baseline values 5-25 HU, no significant contrast enhancement after intravenous administration of contrast material, with septa and no solid components. Otherwise another second-level method, i.e., MR imaging can be employed. A biloma will show heterogeneous signal intensity on T1-weighted sequences and homogeneously hyperintense signals on T2-weighted sequences. MR cholangiography with liver-specific contrast agent (diethylene triamino-pentaacetic acid/gadolinium-ethoxybenzyl-diethylenetriamine pentaacetic acid-Gd-EOB-DTPA) can depict the anatomy of the biliary system and the relationship with the biloma [5]. In the presence of active bile leak, a biloma will present delayed contrast material filling. Some studies have furthermore shown that the same diagnostic result can be obtained using contrast-enhanced US (CEUS) which can show bile leakage and identify the site of rupture after administration of contrast agent directly into the biliary system [7, 8]. However, definitive diagnosis is provided by drainage and biochemical analysis of the fluid.

Spontaneous reabsorption of a biloma of $4 \mathrm{~cm}$ in diameter or greater is rare. The presence of biloma may cause bile peritonitis or development of severe complications (sepsis, abscess, pancreatitis, respiratory failure, gastrointestinal bleeding, necrotizing fasciitis, pulmonary embolism, myocardial infarction), so prompt treatment is recommended. If there is no communication between the biloma and the biliary system and no concomitant local diseases, percutaneous drainage and antibiotic therapy will be sufficient. In the remaining cases, specific surgery is required.

Conflict of interests Valeria Della Valle, Esmeralda Eshja and Emilio Maria Bassi declare that they have no conflict of interest related to this paper. 
Informed consent All procedures followed were in accordance with the ethical standards of the responsible committee on human experimentation (institutional and national) and with the Helsinki Declaration of 1975 , as revised in 2000 . The patient provided written informed consent to the publication of this paper and to the inclusion in this article of information and images that could potentially lead to her identification.

Animal studies This article does not contain any studies involving animal subjects performed by any of the authors.

\section{References}

1. Whipple C (1898) A case of traumatic rupture of the liver: formation of cystic swelling containing bile stained fluid. Lancet $1: 719$

2. Gould L, Patel A (1979) Ultrasound detection of extrahepatic encapsulated bile: "biloma". AJR Am J Roentgenol 132:1014-1015
3. Lee CM, Stewart L, Way LW (2000) Postcholecystectomy abdominal bile collections. Arch Surg 135:538-544. doi:10.1001/ archsurg.135.5.538

4. Umashankkar K (2009) An unusual presentation of biloma five years following cholecystectomy: a case report. Cases J 2:8048. doi:10.4076/1757-1626-2-8048. PMCID: PMC2827112

5. Tana C (2013) Sonographic assessment of a suspected biloma: a case report and review of the literature. World $\mathbf{J}$ Radiol 5(5):220-225. doi:10.4329/wjr.v5.i5.220

6. Cólović R (1991) Retroperitoneal biloma secondary to operative common bile duct injury. HPB Surg 3(3):193-197. doi:10.1155/ 1991/39181. PMID:2043516

7. Mao R, Xu EJ, Li K, Zheng RQ (2010) Usefulness of contrastenhanced ultrasound in the diagnosis of biliary leakage following T-tube removal. J Clin Ultrasound 38(1):38-40

8. Ignee A, Baum U, Schuessler G, Dietrich CF (2009) Contrastenhanced ultrasound-guided percutaneous cholangiography and cholangiodrainage (CEUS-PTCD). Endoscopy 41(8):725-726 